\title{
Análisis bibliométrico de la revista 'Farmacéuticos Comunitarios' (2009-2020) (I): indicadores de productividad y autoría
}

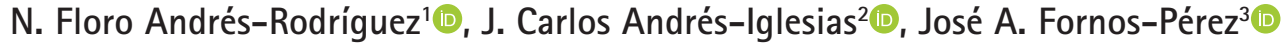 \\ 1. Farmacéutico comunitario en Vigo. Grupo Berbés de Investigación y Docencia. 2. Farmacéutico comunitario en Vigo. Grupo Berbés de \\ Investigación y Docencia. 3. Farmacéutico comunitario en Cangas do Morrazo (Pontevedra). Profesor asociado USC. Grupo Berbés de Investigación \\ y Docencia.
}

\section{PALABRAS CLAVE \\ Bibliometría, indicadores bibliométricos, artículo de revista, farmacéutico comunitario, servicios farmacéuticos comunitarios}

\section{ABREVIATURAS}

CCAA: comunidades autónomas COF: Colegio Oficial de

Farmacéuticos

CGCOF: Consejo General de Colegios Oficiales de Farmacéuticos DE: desviación estándar

GT: grupo de trabajo

IC: índice de cooperación IP: indice de productividad IPL: índice de productividad de Lotka IT: índice de transitoriedad RFC: revista Farmacéuticos Comunitarios

SEFAC: Sociedad Española de Farmacia Clínica, Familiar y Comunitaria.

SEMERGEN: Sociedad Española de Médicos de Atención Primaria

\section{KEYWORDS}

Bibliometrics, bibliometric indicators, journal article, community pharmacist, community pharmacy services

\section{RESUMEN}

Objetivo: analizar, según indicadores bibliométricos, las caracteristicas de la producción científica hallada en los artículos publicados en la revista Farmacéuticos Comunitarios (RFC).

Métodos: diseño: estudio descriptivo retrospectivo de la información bibliométrica obtenida de los artículos publicados en la RFC desde junio 2009 hasta diciembre 2020, consultados en su web.

Indicadores: productividad general (IP), por comunidad autónoma, tipología, número de autores, filiación, índice de cooperación (IC), índice de transitoriedad (IT), índice de productividad de Lotka (IPL).

Resultados: en el período estudiado la RFC publicó 12 volúmenes con 47 números y 297 artículos, con un IP general de 2,5. 148 (49,8\%) fueron artículos de investigación original. 569 autores publicaron al menos un artículo, con un IT del 78,0\% y 6 "grandes productores" ( $\geq 10$ artículos). El IC fue 3,2, con tendencia creciente. Las comunidades autónomas (CCAA) más productivas fueron la Comunidad Valenciana y Galicia. El 70,5\% de los firmantes son farmacéuticos comunitarios, seguidos del 12,1\% de profesores universitarios y el $7 \%$ son médicos. En el $42,8 \%$ de los artículos al menos uno de los autores no es farmacéutico comunitario. SEFAC colaboró con 15 sociedades científicas en la redacción de seis documentos de consenso.

Conclusiones: la mayor parte de los indicadores bibliométricos estudiados en la RFC se encuentran al nivel de otras revistas del área de Ciencias de la Salud, aunque algunos todavía están influidos por el corto recorrido temporal de la revista. Constituye un soporte adecuado, serio y riguroso, para el traslado a la comunidad científica de los resultados de la investigación en su ámbito de actuación.

\section{Bibliometric analysis of the journal 'Farmacéuticos Comunitarios' (2009-2020)}

(I): Indicators of productivity and authorship

\section{ABSTRACT}

Objectives:To analyze, according to bibliometrics indicators, the characteristics of scientific production found in papers published in the journal Farmacéuticos Comunitarios (JFC)

Methods: Design: retrospective descriptive study of the bibliometric information obtained from articles published in the RFC from June 2009 to December 2020, consulted on its website.

Indicators: general productivity (PI), by Autonomous Community, typology, number of authors, affiliation, cooperation index $(\mathrm{Cl})$, transitory index (TI), Lotka productivity index (LPI).

Results: During the period studied, the JFC published 12 volumes with 47 issues and 297 articles, with a general PI of 2.5. 148 (49.8\%) were original research articles. 569 authors published at least one article, with an $\mathrm{TI}$ of $78.0 \%$ and 6 "large producers" ( $\geq 10$ papers). The $\mathrm{Cl}$ was 3.2 , with a growing trend. The most productive AC were the Valencian Community and Galicia. 70.5\% of signatories are community pharmacists, followed by $12.1 \%$ university professors and $7 \%$ medical professors. In $42.8 \%$ of articles at least one of the authors is not a community pharmacist. SEFAC collaborated with 15 scientific societies in the drafting of 6 Consensus Documents.

Conclusions: Most of the bibliometric indicators studied in the JFC are at the level of other journals in the area of Health Sciences, although some are still influenced by the short temporal tour of the journal. It provides adequate, serious and rigorous support for the transfer to the scientific community of research results in its field of action.
Recibido: $11 / 03 / 2021$

Aceptado: 28/06/2021

Disponible online: 06/10/2021
Financiación: ninguna

Conflicto de intereses: ninguno.

Cite este artículo como: Andrés-Rodríguez NF, Andrés-Iglesias JC, Fornos-Pérez JA. Análisis bibliométrico de la revista 'Farmacéuticos Comunitarios' (2009-2020) (I): indicadores de productividad y autoria. Farmacéuticos Comunitarios. 2021 Oct 06; 13 (4): 12-20. doi:10.33620/FC.2173-9218.(2021/Nol13).004.03

Correspondencia: N. Floro Andrés-Rodríguez (fandresr@galicia.com).

ISSN 1885-8619 @SEFAC (Sociedad Española de Farmacia Clínica, Familiar y Comunitaria). Todos los derechos reservados. 


\section{INTRODUCCIÓN}

\section{Difusión de la investigación}

Los farmacéuticos comunitarios españoles realizan investigación sobre su práctica profesional en sus puestos de trabajo, las farmacias comunitarias, bien por iniciativa propia, participando en programas de investigación promovidos por la Organización Colegial o las sociedades científicas, o integrados en grupos de trabajo, independientes, apoyados por dichas instituciones o la Universidad. Muy raramente, aunque algunos existen, llevan a cabo esta investigación en proyectos donde colaboran con las autoridades sanitarias públicas.

La difusión de esta investigación es imprescindible para que tanto resultados como metodología sean conocidos por la comunidad científica y sirvan de base para nuevos estudios que apoyen y consoliden el avance profesional (1).

En los últimos 25 años el número de artículos científicos que difunden investigaciones desarrolladas en el ámbito de la farmacia comunitaria ha aumentado exponencialmente, siguiendo el modelo de Price, que relacionó el incremento de la actividad científica con el aumento de las publicaciones generadas (2). Primero las revistas de la Fundación Pharmaceutical Care España y Pharmacy Practice (antes Seguimiento Farmacoterapéutico), y desde 2009 la nueva etapa de la revista de la Sociedad Española de Farmacia Clínica, Familiar y Comunitaria (SEFAC), Farmacéuticos Comunitarios (RFC), han proporcionado nuevos espacios a disposición de los autores, en esta última con una orientación específica hacia la investigación en el ámbito de la Farmacia Comunitaria y la Atención Primaria de Salud.

La RFC es una publicación multidisciplinar, revisada por expertos de forma anónima (Double-blind peer review), de ámbito nacional, órgano de expresión de SEFAC. Tiene como objetivos promover, fomentar, consolidar y difundir la investigación de calidad en farmacia asistencial en España, Portugal e Hispanoamérica y acercar a los lectores temas profesionales de interés (3). En consecuencia, es el principal soporte para la difusión de la investigación realizada por los socios de SEFAC y al mismo tiempo de las guías, documentos de consenso y resultados de los estudios realizados por la propia Sociedad a través de sus diversos grupos de trabajo (GT).

En la actualidad se edita únicamente en formato electrónico con acceso abierto (Open Access) para lectura y descarga de artículos sin coste para el usuario a través de la web www.farmaceuticoscomunitarios.org.

\section{¿Por qué un análisis bibliométrico?}

La publicación es el producto final de la actividad investigadora, al mismo tiempo que una obligación científica y ética del investigador (4). La bibliometria se centra en el análisis de los valores cuantificables extraídos de la producción y el consumo de la información científica (5). Aplica métodos matemáticos y estadísticos a la literatura de carácter científico y a los autores que la producen con el fin de analizar la calidad de la actividad científica desde el punto de vista de su diseminación. Se apoya en las leyes bibliométricas, que se fundamentan en el comportamiento estadístico regular que muestran a lo largo del tiempo los diferentes elementos que forman parte de las manifestaciones de la actividad científica $(5,6)$.

El análisis bibliométrico permite conocer la evolución de los indicadores que caracterizan la actividad científica generada por los autores y grupos que la difunden mediante los artículos publicados en las revistas científicas acreditadas, y al mismo tiempo contribuye a la mejora de la gestión editorial al generar información de gran utilidad para sus responsables con el fin de corregir posibles desviaciones de las metas planteadas.

\section{Objetivo}

El presente trabajo tiene como objetivo analizar, utilizando criterios bibliométricos, las características de la producción científica que se encuentra en los artículos publicados en la revista Farmacéuticos Comunitarios como instrumento de difusión científico de referencia en el área de la Farmacia Comunitaria española. El consumo de información y otros indicadores se estudiarán en un trabajo posterior.

\section{MATERIAL Y MÉTODOS}

\section{Diseño}

Estudio descriptivo retrospectivo de la información bibliométrica obtenida de los artículos publicados en la RFC desde junio de 2009 hasta diciembre de 2020.

\section{Fuente de obtención de los datos}

La información se obtuvo de la consulta en la web de la revista, donde se encuentra disponible el histórico de números publicados. Se exceptuaron los números dedicados a las comunicaciones a congresos, ya que se podrian producir duplicidades al ser publicados posteriormente como artículos.

\section{Criterios de inclusión}

Se registraron todos los artículos publicados, aunque para ciertos análisis se consideraron tan solo los artículos originales, entendidos como tales aquellos que presentan resultados procedentes de investigaciones propias $(7,8)$, no publicados anteriormente y que se sometieron al proceso de revisión por pares.

\section{Indicadores analizados}

Las herramientas utilizadas para el análisis fueron los indicadores bibliométricos, que nos proporcionan la información sobre las características de la actividad científica que se refleja en los artículos publicados en la revista. 


\section{Indicadores generales de producción}

Se estudió el número y tipología documental de los artículos, su evolución y tendencias en el periodo analizado. El índice de productividad (IP) corresponde al logaritmo del número de artículos. La producción por comunidad autónoma se determinó tomando como criterio la que corresponde al primer autor. Se calculó el número de artículos por farmacia en relación con las existentes en la comunidad autónoma. La información del número de farmacias se obtuvo de la página web del Consejo General de Colegios Oficiales de Farmacéuticos (9).

\section{Indicadores de productividad de autores}

Número de autores por publicación, índice de cooperación (IC) (cociente entre el número de autores y el número de artículos), índice de transitoriedad (IT) (porcentaje de autores ocasionales con respecto al total de autores). Se comprobó el ajuste de la productividad de los autores a la ley de Lotka, según la que la mayoría de los trabajos se deben a unos pocos autores $(5,10)$. El índice de productividad de Lotka (IPL) (logaritmo decimal del número de artículos publicados), agrupa a los autores según su ratio de productividad en: pequeños productores, con una sola publicación $(I P L=0)$, medianos productores, con entre 2 y 9 artículos publicados $(\mathrm{IPL}=0-0,9)$ y grandes productores, con 10 o más trabajos publicados $(I P L \geq 1)(5,11)$.

\section{Análisis de los resultados}

El registro primario de los datos obtenidos de la revisión se realizó en tablas de MSExcel ${ }^{\circledR}$ y para su análisis se utilizó el programa estadístico G-Stat $2.0^{\circledR}$. En el estudio descriptivo de los indicadores, los datos cualitativos se expresan como porcentajes y los cuantitativos, según los casos, como moda o media $(m) \pm$ desviación estándar $(D E)$ y rango. Los límites de confianza se calcularon al 95\%, utilizando las pruebas de t de Student para variables cuantitativas con distribución normal y Mann-Whitney para variables cuantitativas con distribución no normal. La significación estadística se fijó en $p<0,05$.

La calidad de la información se comprobó mediante la doble introducción de los datos, consultando en caso necesario los artículos originales para la aclaración de las discrepancias.

\section{RESULTADOS}

\section{Estructura de la revista}

Farmacéuticos Comunitarios publica cuatro números anuales, con periodicidad trimestral, un suplemento bianual con las comunicaciones al Congreso Nacional de Farmacéuticos Comunitarios correspondiente y, desde 2019 otro suplemento, en este caso anual, dedicado al Congreso Nacional Médico-Farmacéutico SEMERGEN-SEFAC.

\section{Indicadores generales de producción}

En el período analizado (2009-2020) la RFC publicó 12 volúmenes con 47 números (el año inicial, 2009, se publicaron 3 números) y 297 artículos, con una media por volumen anual de 24,8 $(3,7)(20-31)$, un máximo de 31 artículos en 2010 y un índice de productividad general de 2,5. De ellos, 148 (49,8\%) fueron artículos de investigación original (originales + casos clínicos), con un índice de productividad de 2,2. En la tabla 1 se muestra la distribución anual de los artículos en función de su tipología

Tabla 1 Distribución de los artículos en función de su tipología

\begin{tabular}{|c|c|c|c|c|c|c|c|c|c|c|c|c|c|}
\hline Tipo de artículo & 2009 & 2010 & 2011 & 2012 & 2013 & 2014 & 2015 & 2016 & 2017 & 2018 & 2019 & 2020 & Total \\
\hline Editoriales & 5 & 4 & 6 & 5 & 4 & 5 & 4 & 4 & 4 & 5 & 4 & 4 & 54 \\
\hline Originales & 9 & 11 & 9 & 7 & 8 & 14 & 12 & 8 & 9 & 13 & 10 & 10 & 120 \\
\hline Proyectos de investigación & 1 & & 2 & 1 & 1 & 1 & 1 & & & 1 & & 1 & 9 \\
\hline Casos clínicos & 4 & 4 & 2 & & 2 & 1 & & 2 & 4 & 4 & 2 & 3 & 28 \\
\hline Revisiones & 4 & 3 & 5 & 4 & 6 & 1 & 2 & 3 & 1 & & 1 & & 30 \\
\hline Cartas al director & 2 & 2 & 2 & 1 & 1 & & & 0 & 1 & & & & 9 \\
\hline $\begin{array}{l}\text { Documentos, protocolos y } \\
\text { guías clínicas de SEFAC }\end{array}$ & 1 & 1 & 1 & 1 & 1 & 1 & 1 & 3 & & & & & 10 \\
\hline Documentos de consenso & & 2 & 1 & & 1 & 1 & & 1 & & & & & 6 \\
\hline $\begin{array}{l}\text { Colaboraciones, artículos } \\
\text { especiales y de opinión }\end{array}$ & 2 & 4 & 2 & 6 & 3 & 2 & 1 & 2 & 2 & 1 & 4 & 2 & 31 \\
\hline Totales anuales & 28 & 31 & 30 & 25 & 27 & 26 & 21 & 23 & 21 & 24 & 21 & 20 & 297 \\
\hline Artículos por número & 9,3 & 7,8 & 7,5 & 6,3 & 6,8 & 6,5 & 5,3 & 5,8 & 5,3 & 6,0 & 5,3 & 5,0 & 6,4 \\
\hline
\end{tabular}




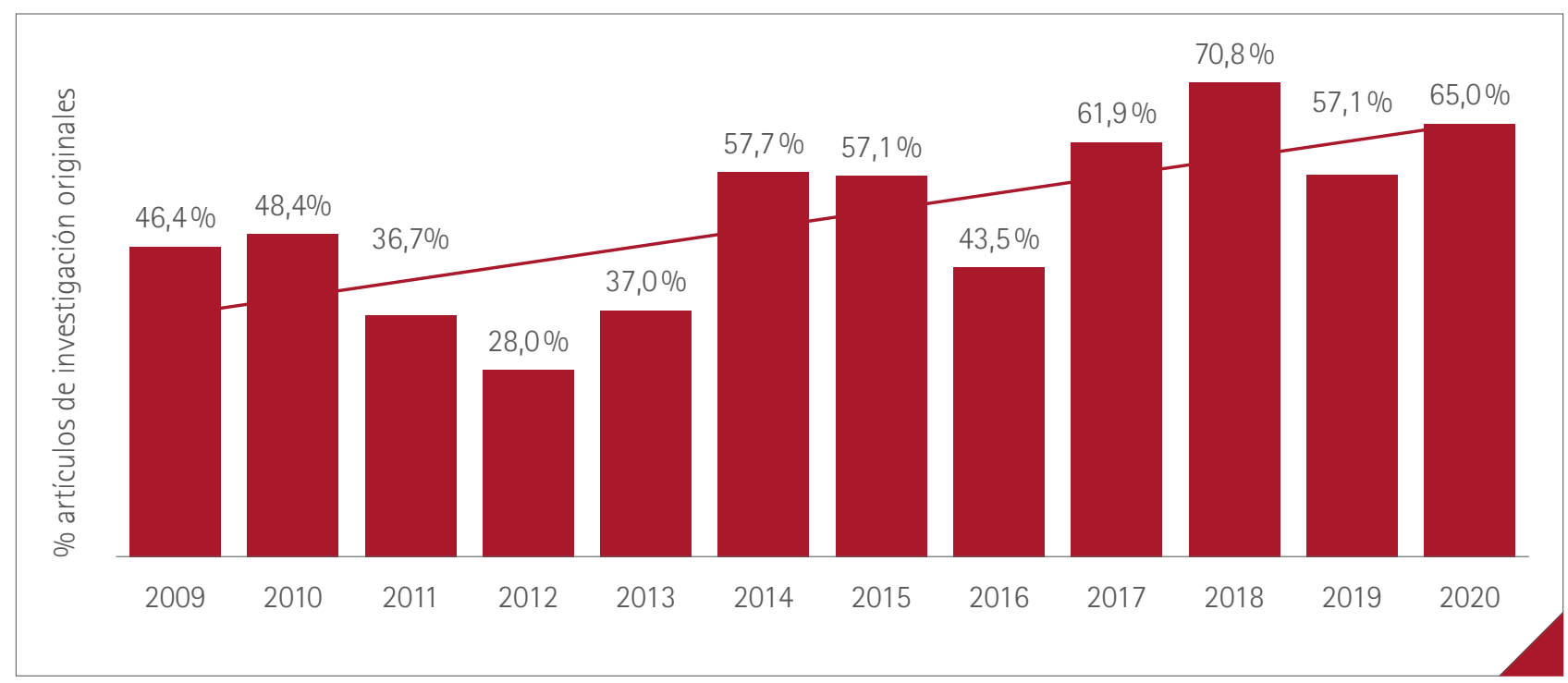

Figura 1 Evolución y tendencia del porcentaje de artículos de investigación originales

y en la figura 1 la evolución y tendencia del porcentaje de artículos originales sobre el total de artículos publicados, con un aumento significativo en el período estudiado $(p<0,01)$.

La media de artículos por número es de $6,4(D E=1,3)$ $(5,0-9,3)$, con un descenso desde los primeros años, que parece haberse estabilizado en los últimos 6 años $(p=$ 0,275).

\section{Indicadores de productividad de autores}

El número total de autores y coautores que han publicado al menos un artículo en la RFC es de 569, de los cuales $444(78,0 \%)$ son "pequeños productores" al figurar como autores en un solo artículo (índice de transitoriedad= $78,0 \%$ ).

La distribución de los autores en función del número de artículos publicados se refleja en la tabla 2. En la última

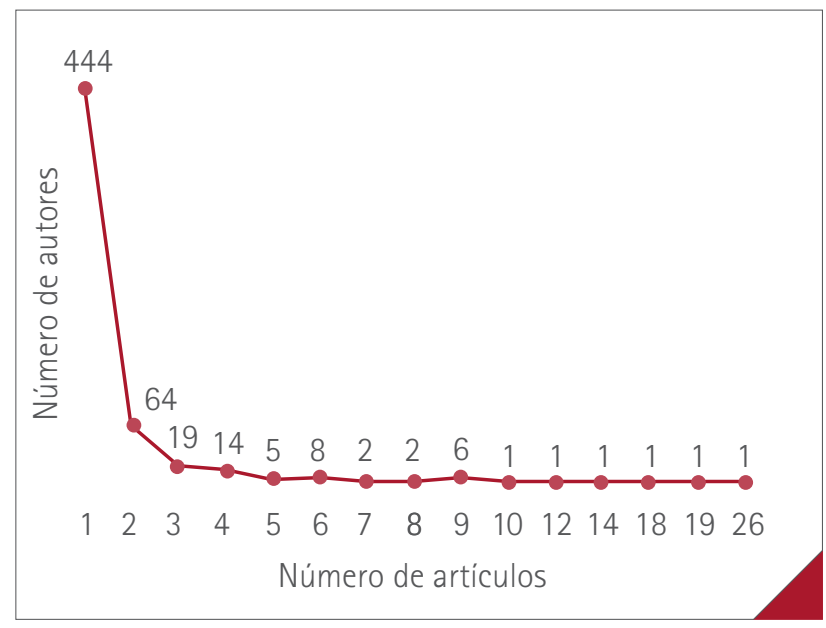

Figura 2 Representación de la productividad de cada autor en aplicación de la ley de Lotka
Tabla 2 Distribución de autores en función del número de artículos publicados

\begin{tabular}{|c|c|c|c|c|}
\hline $\begin{array}{c}\mathrm{N}^{\circ} \\
\text { artículos }\end{array}$ & $\begin{array}{c}\mathrm{N}^{\circ} \\
\text { autores }\end{array}$ & $\%$ & $\begin{array}{c}\% \\
\text { acumulado }\end{array}$ & $\begin{array}{c}\text { IPL de } \\
\text { Lotka }\end{array}$ \\
\hline 1 & 444 & $78,0 \%$ & $100,0 \%$ & 0,00 \\
\hline 2 & 64 & $11,2 \%$ & $22,1 \%$ & 0,30 \\
\hline 3 & 19 & $3,3 \%$ & $10,9 \%$ & 0,48 \\
\hline 4 & 14 & $2,5 \%$ & $7,6 \%$ & 0,60 \\
\hline 5 & 5 & $0,9 \%$ & $5,1 \%$ & 0,70 \\
\hline 6 & 8 & $1,4 \%$ & $4,2 \%$ & 0,78 \\
\hline 7 & 2 & $0,4 \%$ & $2,8 \%$ & 0,85 \\
\hline 8 & 2 & $0,4 \%$ & $2,5 \%$ & 0,90 \\
\hline 9 & 6 & $1,1 \%$ & $2,1 \%$ & 0,95 \\
\hline 10 & 1 & $0,2 \%$ & $1,1 \%$ & 1,00 \\
\hline 12 & 1 & $0,2 \%$ & $0,9 \%$ & 1,08 \\
\hline 14 & 1 & $0,2 \%$ & $0,7 \%$ & 1,15 \\
\hline 18 & 1 & $0,2 \%$ & $0,5 \%$ & 1,26 \\
\hline 19 & 1 & $0,2 \%$ & $0,4 \%$ & 1,28 \\
\hline 26 & 1 & $0,2 \%$ & $0,2 \%$ & 1,41 \\
\hline & 569 & $100,0 \%$ & $100,0 \%$ & \\
\hline
\end{tabular}

columna, el índice de productividad de Lotka muestra que el número de "grandes productores" (IPL $\geq 1)$ es de $6(1,0 \%)$ y el de "medianos productores" (IPL >0-<1) de $120(21,1 \%)$. En la figura 2 se representa la curva correspondiente a la ley de Lotka aplicada a la productividad de los autores de artículos publicados en la RFC. 


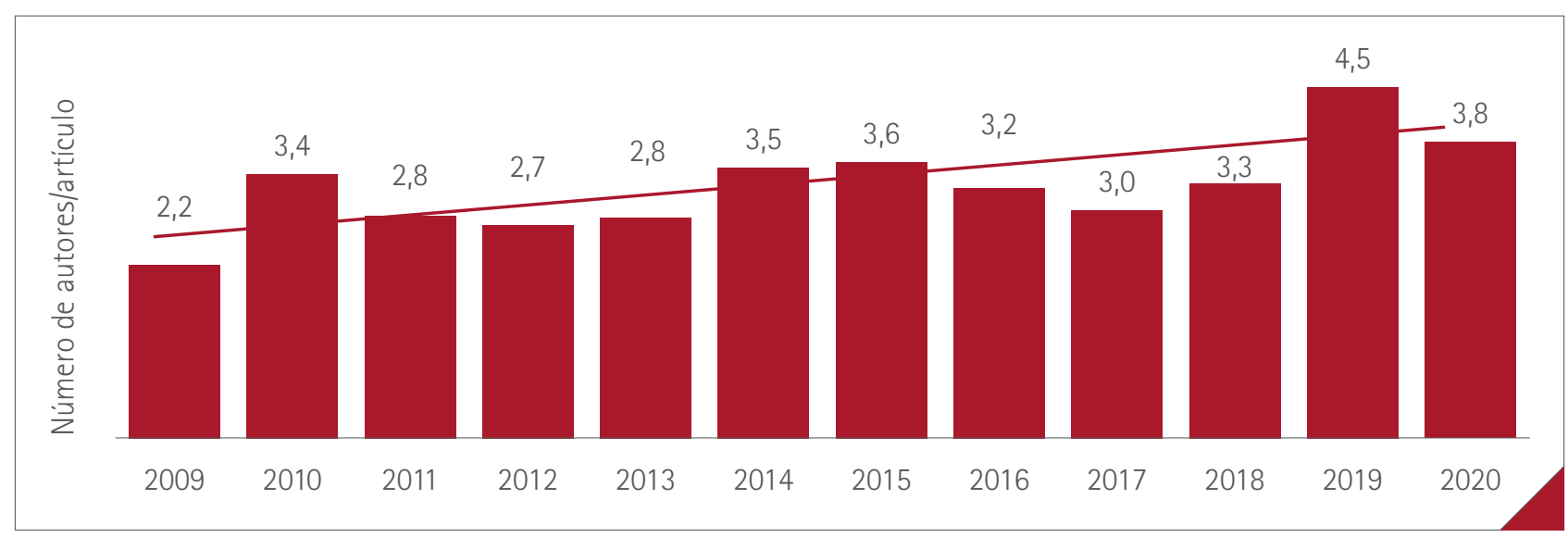

Figura 3 Evolución del número medio de autores/artículo por volumen (año).

El número medio de autores por artículo (índice de cooperación) (se excluyeron 7 artículos de autoría institucional, cuyos autores no se mencionaban) fue $I C=3,2(D E=2,4)$ (1-14), con una moda (número más frecuente de autores) de 1.

La distribución del número medio de autores/artículo (IC) por volumen y su tendencia se muestra en la figura 3.

En la tabla 3 se muestra el número de autores por tipo de artículo. El índice de cooperación medio en los artículos de investigación original (originales y casos clínicos) es de 3,9 $(\mathrm{DE}=2,3)(1-14)$ (moda 3$)$ vs $2,4(2,2)$ (1-11), (moda 1); $p<0,01$.

En la tabla 4 se refleja la producción por comunidad autónoma. Se publicó también un artículo de cada uno de los siguientes paises: Austria, Portugal, Argentina, Costa Rica y México.
Tabla 3 Número de autores/artículo (IC) en función del tipo de artículo

\begin{tabular}{|l|c|}
\hline Tipo de artículo & $\begin{array}{c}\text { No autores } \\
\text { m (DE) (Rango) (moda) }\end{array}$ \\
\hline Editorial & $1,2(0,4)(1-2)(1)$ \\
\hline Original & $4,3(2,3)(1-14)(6)$ \\
\hline Proyectos de investigación & $5,9(1,5)(3-8)(6)$ \\
\hline Casos clínicos & $2,4(1,2)(1-5)(1)$ \\
\hline Revisión & $2,6(1,7)(1-6)(1)$ \\
\hline Cartas al director & $1,6(1,0)(1-4)(1)$ \\
\hline $\begin{array}{l}\text { Documentos, protocolos y } \\
\text { guías de SEFAC }\end{array}$ & $5,8(2,0)(2-9)(6)$ \\
\hline Documentos de consenso & $6,8(1,8)(4-8)(8)$ \\
\hline $\begin{array}{l}\text { Colaboraciones, artículos } \\
\text { especiales y opinión }\end{array}$ & $1,9(2,1)(1-11)(1)$ \\
\hline
\end{tabular}

Tabla 4 Distribución geográfica de los artículos publicados por comunidad autónoma

\begin{tabular}{|l|c|c|c|c|c|c|c|c|c|c|c|c|c|c|}
\hline & 2009 & 2010 & 2011 & 2012 & 2013 & 2014 & 2015 & 2016 & 2017 & 2018 & 2019 & 2020 & Total & $\begin{array}{c}\text { No art./ } \\
\text { farmacia }\end{array}$ \\
\hline Melilla & & & & & & & & & 1 & & & & 1 & 0,040 \\
\hline Navarra & & 1 & 1 & & & & & & & & & & 2 & 0,003 \\
\hline Canarias & & & & & 1 & & & & 1 & & & 1 & 3 & 0,004 \\
\hline Extremadura & & & & & & & 1 & 1 & & & 1 & 1 & 4 & 0,006 \\
\hline Baleares & & & 2 & & & & 1 & 1 & 1 & 2 & & & 7 & 0,016 \\
\hline Castilla-La Mancha & & & & 1 & & & & 1 & 2 & 1 & 1 & 1 & 7 & 0,006 \\
\hline Castilla y León & 1 & & & & 2 & 3 & & & & & 1 & & 7 & 0,004 \\
\hline Región de Murcia & 1 & & & & 1 & 1 & & & 1 & 1 & 1 & 2 & 8 & 0,014 \\
\hline Aragón & 3 & & 1 & 1 & 2 & 2 & & 1 & 2 & 1 & 1 & 1 & 15 & 0,020 \\
\hline País Vasco & & 4 & 3 & 3 & 3 & 1 & & & 1 & & & 2 & 17 & 0,020 \\
\hline Cataluña & 1 & 3 & 1 & 5 & 4 & 4 & 3 & 3 & 2 & & 1 & 1 & 28 & 0,009 \\
\hline Andalucia & 5 & 6 & 4 & 3 & 3 & 3 & 5 & 1 & 2 & 1 & 1 & 3 & 37 & 0,010 \\
\hline C. de Madrid & 3 & 6 & 11 & 3 & 2 & 2 & 3 & 2 & 1 & 2 & 3 & 3 & 41 & 0,014 \\
\hline Galicia & 7 & 4 & 4 & 5 & 3 & 3 & 3 & 7 & 3 & 5 & 4 & 1 & 49 & 0,036 \\
\hline C. Valenciana & 3 & 5 & 2 & 3 & 6 & 6 & 4 & 1 & 1 & 8 & 6 & 5 & 50 & 0,021 \\
\hline & 24 & 29 & 29 & 24 & 27 & 25 & 20 & 18 & 18 & 21 & 20 & 21 & 276 & \\
\hline
\end{tabular}


Tabla 5 Indicadores relativos a la filiación de los autores (las cifras entre paréntesis son \% sobre autores y sobre artículos)

\begin{tabular}{|c|c|c|c|c|c|c|c|c|c|c|c|c|c|}
\hline Indic & 2009 & 2010 & 011 & 012 & 013 & 014 & 015 & 2016 & 2017 & 2018 & 2019 & 2020 & Totales \\
\hline & 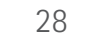 & 31 & 29 & 25 & 8 & 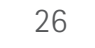 & 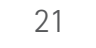 & 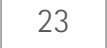 & 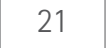 & 24 & 21 & 20 & 297 \\
\hline & & תח & 79 & & 70 & & & & & & 94 & 76 & 916 \\
\hline & $\begin{array}{c}51 \\
(83,6)\end{array}$ & $\begin{array}{c}64 \\
(65,3)\end{array}$ & $\begin{array}{c}50 \\
(63,3)\end{array}$ & $\begin{array}{c}51 \\
(78,5)\end{array}$ & $\begin{array}{c}51 \\
(65,4)\end{array}$ & $\begin{array}{c}59 \\
(66,3)\end{array}$ & $\begin{array}{c}49 \\
(68,1)\end{array}$ & $\begin{array}{c}47 \\
(73,4)\end{array}$ & $\begin{array}{c}47 \\
(75,8)\end{array}$ & $\begin{array}{c}53 \\
(67,9)\end{array}$ & $\begin{array}{c}66 \\
(70,2)\end{array}$ & $\begin{array}{c}58 \\
(76,3)\end{array}$ & $(70,5)$ \\
\hline 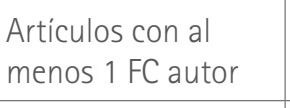 & $\begin{array}{c}26 \\
(92,9) \\
\end{array}$ & $\begin{array}{c}26 \\
(83,9)\end{array}$ & 27 & 23 & $\begin{array}{c}25 \\
(89,3)\end{array}$ & $\begin{array}{c}25 \\
(96,2)\end{array}$ & $\begin{array}{c}19 \\
(90,5)\end{array}$ & $\begin{array}{c}19 \\
(82,6)\end{array}$ & $\begin{array}{c}20 \\
(95,2)\end{array}$ & $\begin{array}{c}22 \\
(91,7)\end{array}$ & $\begin{array}{c}20 \\
(95,2)\end{array}$ & $\begin{array}{c}20 \\
(100,0)\end{array}$ & $\begin{array}{c}272 \\
(91,6)\end{array}$ \\
\hline $\begin{array}{l}\text { Artículos con al } \\
\text { menos } 1 \text { autor no FC }\end{array}$ & $\begin{array}{c}5 \\
(17,9)\end{array}$ & $\begin{array}{c}18 \\
(58,1)\end{array}$ & $\begin{array}{c}13 \\
(44,8)\end{array}$ & $\begin{array}{c}7 \\
(28,0)\end{array}$ & $\begin{array}{c}12 \\
(42,9)\end{array}$ & $\begin{array}{c}10 \\
(38,5)\end{array}$ & $\begin{array}{c}12 \\
(57,1)\end{array}$ & $\begin{array}{c}10 \\
(43,5)\end{array}$ & $\begin{array}{c}8 \\
(38,1)\end{array}$ & $\begin{array}{c}12 \\
(50,0)\end{array}$ & $\begin{array}{c}12 \\
(57,1)\end{array}$ & $\begin{array}{c}8 \\
(40,0)\end{array}$ & $\begin{array}{c}127 \\
(42,8)\end{array}$ \\
\hline
\end{tabular}

FC: farmacéutico comunitario.

Tabla 6 Filiaciones declaradas por los autores

\begin{tabular}{|l|c|l|c|}
\hline Titulación/Ámbito & $\begin{array}{c}\text { Firmas } \\
\mathbf{n}(\%)\end{array}$ & Titulación/Ámbito & $\begin{array}{c}\text { Firmas } \\
n(\%)\end{array}$ \\
\hline Farmacéutico comunitario & $646(70,5)$ & Departamento Técnico de CGCOF & $5(0,5)$ \\
\hline Profesor de universidad & $111(12,1)$ & Junta Gobierno COF & $3(0,4)$ \\
\hline Médico & $64(7,0)$ & Veterinario & $2(0,2)$ \\
\hline Alumno de Farmacia & $12(1,3)$ & Biólogo & $1(0,1)$ \\
\hline Farmacéutico hospitalario & $10(1,1)$ & Farmacéutico de AP & $1(0,1)$ \\
\hline Técnico de investigación & $10(1,1)$ & Farmacéutico militar & $1(0,1)$ \\
\hline Departamento Técnico de COF & $9(1,0)$ & Farmacéutico de la Administración & $1(0,1)$ \\
\hline Abogado & $8(0,9)$ & Alumno de Medicina & $1(0,1)$ \\
\hline Técnico en Farmacia & $8(0,9)$ & Psicólogo & $1(0,1)$ \\
\hline Farmacéutico consultor & $6(0,7)$ & Periodista & $1(0,1)$ \\
\hline Farmacéutico de la industria & $5(0,5)$ & Matemático & $1(0,1)$ \\
\hline Auxiliar de Farmacia & $5(0,5)$ & Sociólogo & \\
\hline
\end{tabular}

CGCOF: Consejo General de Colegios Oficiales de Farmacéuticos; COF: Colegio Oficial de Farmacéuticos; AP: Atención Primaria.

\section{Indicadores de filiación de autores}

El total de autores que figuran en los 297 artículos publicados en la revista en el período de estudio es de 916 , de los que $646(70,5 \%)$ son farmacéuticos comunitarios. En 272 $(91,6 \%)$ figura al menos un farmacéutico comunitario y en $127(42,8 \%)$ al menos uno de los autores declara otra titulación o ámbito de ejercicio.

En la tabla 5 se presentan los principales indicadores relativos a la filiación de los autores.

Las filiaciones declaradas por la totalidad de los autores se presentan en la tabla 6 .
Las universidades de procedencia de autores con esta filiación que con mayor frecuencia firman los artículos son: CEU Universidad Cardenal Herrera, 17 (5,7\%); Granada, 15 (5,1\%); Sevilla, 11 (3,7\%); Universidad del País Vasco, 9 (3,0\%); Valencia, 8 (2,7\%). En 9 ocasiones se trata de universidades del extranjero: Austria, Portugal, Argentina, Costa Rica y México.

En $62(20,9 \%)$ artículos la autoría es asumida por grupos de trabajo o estudio: GT de SEFAC, 26 (8,8\%); GT de universidades, 19 (6,4\%); GT de colegios de farmacéuticos, 17 (5,7\%).

SEFAC colaboró con 15 sociedades científicas en la redacción de seis documentos de consenso (tabla 7). 
Tabla 7 Sociedades científicas con las que colaboró SEFAC en la elaboración de Documentos de Consenso

\begin{tabular}{|l|c|}
\hline Sociedad Científica & $\begin{array}{c}\text { No de } \\
\text { colaboraciones }\end{array}$ \\
\hline $\begin{array}{l}\text { Sociedad Española de Médicos de } \\
\text { Atención Primaria (SEMERGEN) }\end{array}$ & 3 \\
\hline $\begin{array}{l}\text { Sociedad Española de Medicina de } \\
\text { Familia y Comunitaria (semFYC) }\end{array}$ & 2 \\
\hline $\begin{array}{l}\text { Sociedad Española de Medicina Interna } \\
\text { (SEMI) }\end{array}$ & 2 \\
\hline Sociedad Española de Diabetes (SED) & 2 \\
\hline $\begin{array}{l}\text { Sociedad Española de Arteriosclerosis } \\
\text { (SEA) }\end{array}$ & 1 \\
\hline Sociedad Española de Cardiología (SEC) & 1 \\
\hline $\begin{array}{l}\text { Sociedad Española de Endocrinología y } \\
\text { Nutrición (SEEN) }\end{array}$ & 1 \\
\hline $\begin{array}{l}\text { Sociedad Española de Médicos Generales } \\
\text { y de Familia (SEMG) }\end{array}$ & 1 \\
\hline Sociedad Española de Nefrología (SEN) & 1 \\
\hline $\begin{array}{l}\text { Sociedad Española de Hipertensión- } \\
\text { Liga Española para la Lucha contra la } \\
\text { Hipertensión Arterial (SEH-LELHA) }\end{array}$ & 1 \\
\hline Fundación Pharmaceutical Care España & 1 \\
\hline $\begin{array}{l}\text { Red de Grupos de Estudio de la Diabetes } \\
\text { en Atención Primaria de la Salud } \\
\text { (redGDPS) }\end{array}$ & 1 \\
\hline $\begin{array}{l}\text { Sociedad Española de Geriatría y } \\
\text { Gerontología (SEGG) }\end{array}$ & 1 \\
\hline $\begin{array}{l}\text { Sociedad Española de Medicina Geriátrica } \\
\text { (SEMEG) }\end{array}$ & 1 \\
\hline $\begin{array}{l}\text { Sociedad Española de Neumología y } \\
\text { Cirugia Torácica (SEPAR) }\end{array}$ & 1 \\
\hline & 1 \\
\hline
\end{tabular}

\section{DISCUSIÓN}

Desde su inicio en 2009 la revista Farmacéuticos Comunitarios cumple con su compromiso editorial publicando puntualmente sus cuatro números anuales con periodicidad trimestral. La publicación de los suplementos bienales correspondientes a los resúmenes de comunicaciones al Congreso Nacional de Farmacéuticos Comunitarios y los anuales del Congreso Médico-Farmacéutico SEMERGEN-SEFAC se suma a la productividad científica de la RFC, aunque por criterios de evitar posibles duplicidades al publicarse posteriormente artículos incluyendo contenidos de dichas comunicaciones, no se han contabilizado en este análisis bibliométrico, criterio que es seguido en otros equivalentes (12-15).
La accesibilidad a los artículos a través de la web de la revista para su revisión y tabulación de los indicadores acordados ha sido total y rápida, sin ningún error en los enlaces.

\section{Indicadores de productividad científica}

En el período analizado se publicaron en la RFC 297 artículos con una media anual de 24,8 . Prácticamente la mitad $(49,8 \%)$ fueron artículos de investigación original. Los índices de productividad son, por lo tanto, de 2,5 el general y de 2,2 el de originales, similares a los de revistas próximas $(12,15,16)$, menor que una (17) y mayor que otra (18).

Se observa una tendencia decreciente en el número total de artículos, que se estabilizó a partir de 2014, y paralelamente un incremento en el número y porcentaje de originales, situándose alrededor del $64 \%$ en los últimos cuatro años. Es consecuencia del estímulo que la revista viene prestando a la investigación en el ámbito comunitario, la intensa actividad formativa de SEFAC, la creación de grupos de trabajo y la posibilidad que proporcionan los congresos para un primer paso en la difusión. Se acerca, pero aún está lejos, del encontrado por López-Pintor et al. (19) en su revisión de las publicaciones existentes en MEDLINE sobre farmacia comunitaria.

Dentro de la tipología, a grandes rasgos coincidente con la encontrada en otras publicaciones biomédicas, existe un número considerable de documentos, protocolos y guías clínicas de SEFAC, así como documentos de consenso elaborados junto con otras sociedades científicas y dados a conocer en ocasiones simultáneamente. Esta labor de difusión y formación de los socios es de vital importancia en profesiones sanitarias, donde los objetivos terapéuticos, los procedimientos de actuación y los criterios de derivación e interconsulta deben consensuarse y compartirse en beneficio de lograr prácticas homogéneas en la atención a los pacientes.

\section{Productividad de los autores}

De los 569 autores que han publicado en la RFC el 78\%, 444, son "pequeños productores", cifra que se denomina también indice de transitoriedad; es decir, que su contribución es de solo un artículo. Tan solo seis autores pueden considerarse "grandes productores", con un IPL $\geq 1$, al aparecer como autores o coautores en 10 o más artículos. No obstante, ambos indicadores son mejores que los encontrados en otros estudios, con IT de 84,8 (13) y 87,8 (18) y 89,0 (20). En un trabajo nuestro referido a un período anterior (1995-2005) (21) el IT fue algo menor $(71,3)$ con un único autor gran productor, posiblemente por encontrarse la investigación en farmacia comunitaria todavía en una fase muy temprana. En otros trabajos $(15,16,19)$ se analiza la productividad institucional, por lo que no podemos establecer comparaciones con la productividad individual de los autores.

El número de autores por artículo (índice de cooperación) es de 3,2 (en los artículos de investigación original es significativamente mayor que en el resto, 3,9), mayor que en 
dos casos $(20,22)$, próximo al encontrado por López Pintor et al. (19), pero menor que el hallado por nosotros en el estudio referido (21). También menor que en otros ámbitos $(12,14,17,23)$, más acostumbrados al trabajo colaborativo, que forman equipos en su trabajo habitual en instituciones generalmente de carácter público. A pesar de que hay un incremento constante del índice de cooperación a lo largo del tiempo, el bajo número de autores por artículo refleja las características del ejercicio diario del farmacéutico comunitario, en el que no es frecuente grupos extensos de profesionales en los que es más fácil llevar a cabo proyectos de investigación y la "juventud" de estas actividades en las farmacias, tal como observan López-Pintor et al. (19).

\section{Procedencia geográfica}

La procedencia geográfica de los artículos es española en abrumadora mayoría, tan solo cinco países, dos europeos (Austria y Portugal) y tres iberoamericanos (Argentina, Costa Rica y México) aportan un artículo cada uno. Creemos que puede deberse a las diferencias existentes en los modelos de farmacia, especialmente en Iberoamérica, lo que podria restar interés a los profesionales de estos países por publicar en la RFC.

En cuanto a las comunidades autónomas (CCAA) de procedencia de los artículos, Comunidad Valenciana, Galicia y la Comunidad de Madrid ocupan los primeros lugares, mientras que cuatro CCAA no aportan ninguno, en una relativa relación con el grado de implantación y número de socios de SEFAC. Galicia y Comunidad Valencia (aparte el caso de Melilla) presentan también el mejor índice de artículos por farmacia. La Comunidad de Madrid y Comunidad Valenciana (12) y la Comunidad de Madrid (14) son las CCAA más productoras en dos revistas de ámbito de la Salud Pública y hospitalario.

\section{Filiación de los autores}

Como era de esperar en una revista que es órgano de expresión de la sociedad científica de los farmacéuticos comunitarios, esta es la filiación de la gran mayoría de los autores que en ella publican. No se trata de una endogamia artificial o selectiva, sino de la idoneidad del medio. El 70,5\% de los firmantes de artículos en la RFC son, por tanto, farmacéuticos comunitarios, pero en el $42,8 \%$ de los artículos al menos uno de los autores pertenece a otra profesión o ámbito de ejercicio. En el 8,4\% de los artículos ninguno de los autores es farmacéutico comunitario. Estas proporciones permanecen constantes a lo largo del período estudiado, lo que indica la apertura de la RFC a la cooperación interdisciplinar y al reflejo en su producción científica de otros puntos de vista, aunque siempre relacionados con el ámbito e interés de la investigación en farmacia comunitaria.

Los profesores de universidad son el segundo grupo con mayor proporción de firmas, seguido de los médicos y los alumnos de Farmacia. Las universidades de procedencia de aquellos son a su vez representativas del interés dedicado por sus facultades de Farmacia a la docencia de las necesidades formativas reales y a la capacitación de sus alumnos de grado para desempeñar su ejercicio profesional en las mejores condiciones para el beneficio de la salud de sus pacientes.

En un porcentaje considerable de los artículos la autoría es asumida por grupos de trabajo o estudio. Entre ellos en primer lugar los GT de SEFAC, pero también de universidades y Colegios Oficiales de Farmacéuticos. En ambos casos, la aportación del rigor y la experiencia investigadora de este grupo de firmas a la RFC incrementa el valor científico de los trabajos en ella publicados.

En la RFC se refleja la colaboración con otras 15 sociedades científicas en la redacción de seis documentos de consenso, siendo el mayor número de colaboraciones con la Sociedad Española de Médicos de Atención Primaria, SEMERGEN, con tres documentos, Esta colaboración pone de manifiesto una presencia activa de miembros de SEFAC en las tareas de información, estudio, contraste y redacción de dichos documentos. Su contribución como especialistas naturales en el medicamento permite completarlos con el punto de vista cualificado del responsable de la fase final del fármaco, incluyendo el uso por los pacientes.

Consideramos que esta amplia diversidad de filiaciones que encontramos en las firmas de los artículos publicados en la RFC es muy positiva y muestra su voluntad de apertura multidisciplinar, en la que se deberá perseverar con el fin de atraer también a un mayor número de autores de otros países.

Entre las limitaciones que podemos señalar encontramos en primer lugar el uso de la filiación del primer autor como procedencia del artículo, lo cual, en el caso de colaboraciones entre autores de distinta procedencia podría introducir un cierto sesgo. Por otro lado, un indicador como el índice de productividad (logaritmo del número de artículos) es de difícil comparabilidad, ya que podría ser notablemente distinto al calculado si se tuviese en cuenta la dimensión de los períodos analizados, algo que no hemos encontrado advertido en la literatura consultada.

La bibliometría permite conocer de manera objetiva una serie de indicadores sobre las caracteristicas de la producción científica de una revista, un área de conocimiento o un país. Para una revista como Farmacéuticos Comunitarios, este análisis con técnicas bibliométricas de la producción científica reflejada en los artículos publicados es una herramienta de gran utilidad para la toma de decisiones en la gestión organizativa, el establecimiento de prioridades, la asignación de recursos y la planificación de proyectos.

En la revista Farmacéuticos Comunitarios la mayor parte de los indicadores bibliométricos estudiados se encuentran al nivel de los referidos para otras revistas del área de las Ciencias de la Salud, aunque algunos todavía necesitan consolidarse como consecuencia del pequeño recorrido 
temporal de la revista, lo que con toda seguridad se producirá en pocos años. En particular, seguir incrementado el número de artículos originales, mejorar el índice de transitoriedad estimulando la continuidad de los autores en la investigación, mediante formación y herramientas de apoyo, y el de cooperación, favoreciendo los proyectos colaborativos y la formación de grupos de investigación.

La investigación en Farmacia Comunitaria está todavía en fase de expansión, y la RFC sin duda tendrá un papel protagonista, ya que constituye un soporte adecuado, serio y riguroso, para el traslado a la comunidad científica de los resultados de la investigación en las farmacias comunitarias sobre la práctica del ejercicio profesional y la prestación de servicios profesionales farmacéuticos asistenciales a los pacientes que a ellas acuden.

\section{REFERENCIAS BIBLIOGRÁFICAS}

1. Andrés Rodríguez NF. Investigación de resultados en salud en farmacia comunitaria [Lección inaugural del Curso 2015 en la Real Academia de Farmacia de Galicia]. Vigo: RAFG; 2015. Disponible en: https://academiadefarmaciadegalicia.gal/wp-content/uploads/ docs/1482078306wpdm_Lecci\%C3\%B3n\%20Inaugural\%20 Curso\%202015.pdf

2. Price DJS. Network of scientific papers. Science 1965; 149: 510-515. Disponible en: https://garfield.library.upenn.edu/papers/pricenetwor ks1965.pdf

3. Sociedad Española de Farmacia Familiar y Comunitaria. [Internet]. Revista Farmacéuticos Comunitarios: Presentación. [Acceso 25/1/2021]. Disponible en: http://www.farmaceuticoscomunitarios. org/es/presentacion

4. González de Dios J, Moya M, Mateos Hernández MA. Indicadores bibliométricos: caracteristicas y limitaciones en el análisis de la actividad científica. An Esp Pediatr. 1997; 47:235-244 . Disponible en: https://www.aeped.es/sites/default/files/anales/47-3-3.pdf

5. Ardanuy J. Breve introducción a la bibliometría. Barcelona: Universidad de Barcelona; 2012. Disponible en: http://diposit.ub.edu/dspace/ bitstream/2445/30962/1/breve\%20introduccion\%20bibliometria. pdf

6. OECD and SCImago Research Group (CSIC). Compendium of Bibliometric Science Indicators. Paris: OECD; 2016. Disponible en: https:// www.oecd.org/sti/inno/Bibliometrics-Compendium.pdf

7. Mangan R. [Libro en Internet]. Web of Science. Manual de uso. Web of Science Group. 2019. [Acceso 3/2/2021]. Disponible en: https:// www.recursoscientificos.fecyt.es/sites/default/files/spanish_manual_wos_01_03_2019.pdf

8. Day RA. Cómo escribir y publicar trabajos científicos. Washington DC: Organización Panamericana de la Salud; 2005. Pág. 8. Disponible en: https://www3.paho.org/hq/index.php?option=com_content\&tview=articlectid=2234:2010-como-escribir-publicar-trabajos-cientificos\&ttemid $=1894$ \&tlang $=e s$

9. CGCOF. Estadísticas de Colegiados y Farmacias Comunitarias 2019. [Acceso 31/1/21]. Disponible en: https://www.portalfarma.com/Profesionales/infoestadistica/Documents/Estad\%c3\%adsticas-Colegiados-Farmacias-2019.pdf

10. Sancho, R. Indicadores bibliométricos utilizados en la evaluación de la Ciencia y la Tecnología. Rev Esp Doc Cient. 1990; 13(3-4): 842865. Disponible en: http://digital.csic.es/bitstream/10261/23694/1/ SAD_DIG_IEDCyT_Sancho_Revista\%20Espa\%C3\%B1ola\%20de\%20 Documentacion\%20Cientifica 13\%284\%29.pdf
11. Tomás-Górriz V, Tomás-Casterá V. La Bibliometría en la evaluación de la actividad científica. Hosp Domic. 2018;2(4):145-63. doi:10.22585/ hospdomic.v2i4.51

12. Pérez Andrés C, Estrada Lorenzo JM, Villar Álvarez F, Rebollo Rodríguez MJ. Estudio bibliométrico de los artículos originales de la Revista Española de Salud Pública (1991-2000). Parte primera: Indicadores generales. Rev Esp Salud Pública. 2002; 76(6): 659-672. Disponible en: https://scielo.isciii.es/scielo.php?script=sci_arttextetpid=S1135-57272002000600003

13. Estrada Lorenzo JM, Villar Álvarez F, Pérez Andrés C, Rebollo Rodríguez MJ. Estudio bibliométrico de los artículos originales de la Revista Española de Salud Pública (1991-2000). Parte segunda: productividad de los autores y procedencia institucional y geográfica. Rev Esp SaIud Pública. 2003; 77(3): 333-346. Disponible en: https://scielo.isciii. es/scielo.php?script=sci_arttextEtpid=S1135-57272003000300004

14. Tomás Casterá V, Sanz Valero J, Juan-Quilis V, Wanden-Berghe $C$, Culebras JM, García de Lorenzo y Mateos A. Estudio bibliométrico de la revista Nutrición Hospitalaria en el periodo 2001 a 2005: parte 2, análisis de consumo; las referencias bibliográficas. Nutr Hosp. 2008; 23: 541-546. Disponible en: https://www.nutricionhospitalaria.org/ magazines/278/show

15. Sanz-Valero J, Tomás-Casterá V, Tomás-Gorriz V. Estudio bibliométrico de producción y consumo de la revista Farmacia Hospitalaria (2004-2012). Farm Hosp. 2014; 38(1): 1-8. DOI: 10.7399/ FH.2014.38.1.1153. Disponible en: https://revistahad.eu/index.php/ revistahad/article/download/3/4

16. Sanz-Valero J, Tomás-Gorriz V, Morales Suárez-Varela MM. Estudio bibliométrico de la producción científica publicada por la Revista Ars Pharmaceutica en el periodo 2001 a 2013. Ars Pharm. 2014; 55(2): 1-10. Disponible en: https://revistaseug.ugr.es/index.php/ars/article/ download/4488/4391/

17. Sanz-Valero J, Tomás Casterá V, Wanden-Berghe C. Estudio bibliométrico de la producción científica publicada por la Revista Panamericana de Salud Pública/Pan American Journal of Public Health en el período de 1997 a 2012. Rev Panam Salud Publica. 2014; 35(2): 17. 81-8. Disponible en: https://iris.paho.org/bitstream/handle/10665.2/8465/a01v 35n2.pdf? sequence $=1$ EtisAllowed $=\mathrm{y}$

18. Reyes A, Olate $Y$, Godoy C. Análisis bibliométrico de la producción cientifica de la Revista Actividad Física y Ciencias durante el período 2009-2018. Revista Ciencias de la Actividad Física UCM, 2019; 20(2): 1-25. Disponible en: http://revistacaf.ucm.cl/article/view/361/399

19. López-Pintor $E_{1}$ Núñez Cacho-Canales P, Sanz-Valero J. Análisis bibliométrico y temático de la producción científica existente en la base de datos bibliográfica MEDLINE sobre farmacia comunitaria. Pharm Care Esp. 2018; 20(6): 408-428. Disponible en: https:// www.pharmcareesp.com/index.php/PharmaCARE/article/down$\operatorname{load} / 473 / 386$

20. Flores-Fernández C, Aguilera-Eguia R, Saldivia Saldivia AM, Gutiérrez Parra V, Pérez-Galdavini VM, Torres Morera LM. Análisis bibliométrico de la Revista de la Sociedad Española del Dolor: 2007-2016. Rev Soc Esp Dolor 2018; 25(3): 170-177. Disponible en: https://scielo. isciii.es/pdf/dolor/v25n3/1134-8046-dolor-25-03-00170.pdf

21. Andrés Iglesias JC, Andrés Rodríguez NF, Fornos Pérez JA. Community pharmacy-based research in Spain (1995-2005): A bibliometric study. Pharmacy Practice 2007; 5(1): 21-30. Disponible en: https:// scielo.isciii.es/pdf/pharmacy/v5n1/021-030.pdf

22. Corrales-Reyes IE, Fornaris-Cedeño Y, Reyes-Pérez JJ. Análisis bibliométrico de la revista Investigación en Educación Médica. Período 2012-2016. Inv Ed Med. 2018; 7(25): 18-26. doi:10.1016/j. riem.2017.02.003

23. Franco-Paredes K, Díaz-Reséndiz FJ, Pineda-Lozano JE, Hidalgo-Rasmussen CA. Bibliometric analysis of scientific production of Mexican Journal of Eating Disorders, 2010-2014. Revista Mexicana de Trastornos Alimentarios. 2016; 7: 9-16. doi:10.1016/j.rmta.2016.03.001 